\title{
Hot Embossing of Zr-Based Bulk Metallic Glass Micropart Using Stacked Silicon Dies
}

\author{
Zhijing Zhu, ${ }^{1}$ Chuanyun Yi, ${ }^{1}$ Tielin Shi, ${ }^{1}$ Yichun Zhang, ${ }^{1}$ Yang Gao, ${ }^{2}$ and Guanglan Liao ${ }^{1}$ \\ ${ }^{1}$ State Key Laboratory of Digital Manufacturing Equipment and Technology, Huazhong University of Science and Technology, \\ Wuhan 430074, China \\ ${ }^{2}$ Jiangxi Province Key Laboratory of Numerical Control Technology and Application, Jiujiang 332005, China
}

Correspondence should be addressed to Guanglan Liao; guanglan.liao@hust.edu.cn

Received 11 February 2015; Revised 29 May 2015; Accepted 14 June 2015

Academic Editor: Jörg M. K. Wiezorek

Copyright (C) 2015 Zhijing Zhu et al. This is an open access article distributed under the Creative Commons Attribution License, which permits unrestricted use, distribution, and reproduction in any medium, provided the original work is properly cited.

\begin{abstract}
We demonstrated hot embossing of $\mathrm{Zr}_{65} \mathrm{Cu}_{17.5} \mathrm{Ni}_{10} \mathrm{Al}_{7.5}$ bulk metallic glass micropart using stacked silicon dies. Finite element simulation was carried out, suggesting that it could reduce the stress below $400 \mathrm{MPa}$ in the silicon dies and enhance the durability of the brittle silicon dies when using varying load mode ( $100 \mathrm{~N}$ for $60 \mathrm{~s}$ and then $400 \mathrm{~N}$ for $60 \mathrm{~s}$ ) compared with using constant load mode $(200 \mathrm{~N}$ for $120 \mathrm{~s})$. A micropart with good appearance was fabricated under the varying load, and no silicon die failure was observed, in agreement with the simulation. The amorphous state of the micropart was confirmed by differential scanning calorimeter and X-ray diffraction, and the nanohardness and Young's modulus were validated close to those of the as-cast BMG rods by nanoindentation tests. The results proved that it was feasible to adopt the varying load mode to fabricate three-dimensional Zr-based bulk metallic glass microparts by hot embossing process.
\end{abstract}

\section{Introduction}

With superior strength, high hardness, large elasticity, excellent wear, and corrosion resistance, BMGs (Bulk Metallic Glasses) are recognized as promising materials for structure, sensor, wear-resistant, and corrosion-resistance coating [1, 2]. When formed into microparts, their macroscopic brittle nature can be mitigated $[3,4]$, which suggests desirable applications in MEMS (Microelectromechanical Systems).

To fabricate BMG microparts, there are mainly two processes: direct casting and thermoplastic forming [5]. The former casts the molten alloy into a microdie followed by fast cooling to inhibit crystallization [6,7]. This process is usually carried out to form macroscale BMG materials [8,9], which can be used as feedstock for other processes [10]. The latter reheats the BMG feedstock into a viscous metastable supercooled liquid $[11,12]$ and forms it into microdies before crystallization occurring [13], which is also called hot embossing $[14,15]$ or superplastic forming [16, 17]. The hot embossing process is considered to be more controllable compared with direct casting because of relatively long processing time and separation of forming and fast cooling [18] and has been used to fabricate microparts $[19,20]$ and microdies $[20,21]$. For forming microparts by hot embossing process, microdies are necessary. As silicon owns high strength and hardness and can be structured into micro geometry with high precision and low surface roughness by lithography and etching process, it has been widely used for microdies in hot embossing process to form BMG microparts $[19,22]$. However, due to brittleness and strength reduction caused by the etching process, silicon dies failure can be observed [23]. To enhance the durability of the silicon dies, the load in the hot embossing process needs to be optimized. In addition, most of microparts fabricated by the hot embossing process using silicon dies are quasi three-dimensional structures, which cannot satisfy diverse requirements in MEMS [24].

In this work, stacked silicon dies for fabricating threedimensional microparts were prepared by lithography and ICP (Inductively Coupled Plasma) etching process. Simulations and experiments were carried out to optimize the hot embossing process. A three-dimensional BMG microgear 


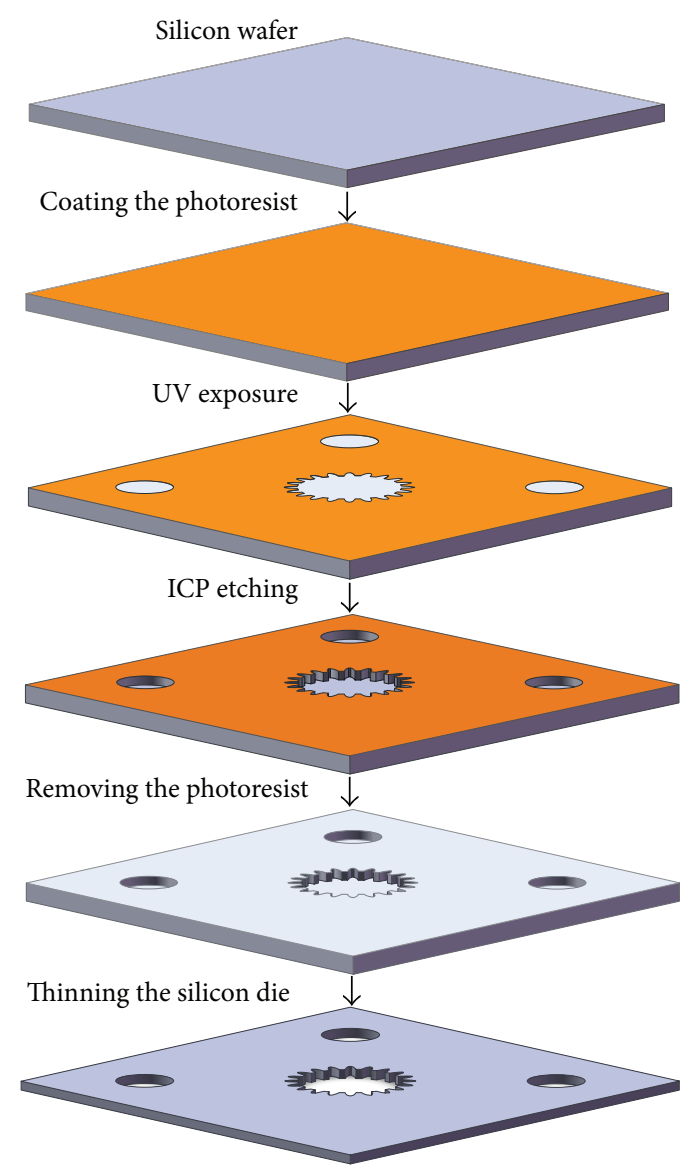

(a)

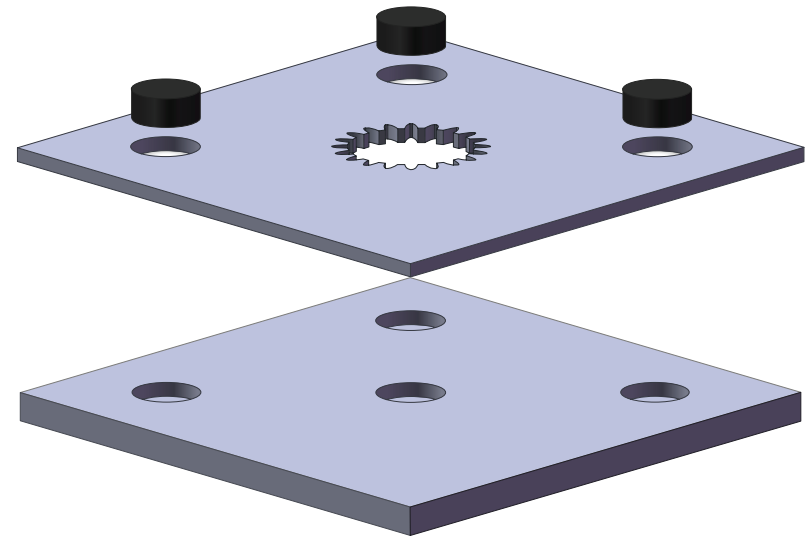

(b)

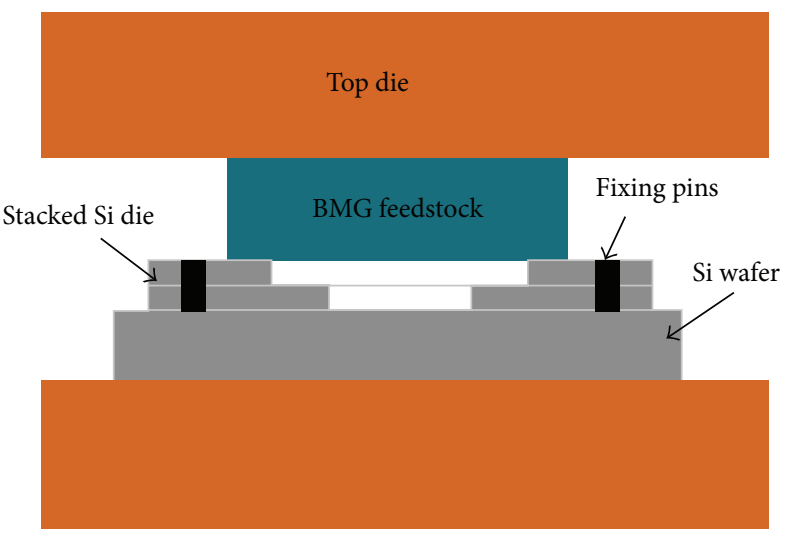

(c)

FIGURE 1: Sketches of silicon dies preparation (a), silicon dies stacking (b), and hot embossing process (c).

with good appearance was hot embossed successfully, its glassy state was detected by DSC (Differential Scanning Calorimeter) and XRD (X-Ray Diffraction), and the mechanical properties were validated by nanoindentation tests.

\section{Experimental}

Figure 1(a) sketched silicon dies preparing process. The silicon wafer was coated with photoresist (AZ9260), exposed for 100 s (MA6, Karl Suss), and followed by developing to transfer patterns onto the photoresist. The exposed region of the silicon wafer was etched to a depth of $300 \mu \mathrm{m}$ by ICP process (Oxford PlasmaLab System 100). After that, the silicon wafer was thinned by mechanical polishing to make the cavity through the die (Ecomet 300/Automet 300, Buehler). The silicon dies were stacked and aligned by pins to assemble dual-layer dies, as shown in Figure 1(b).

A $\mathrm{Zr}_{65} \mathrm{Cu}_{17.5} \mathrm{Ni}_{10} \mathrm{Al}_{7.5}$ BMG rod with $3 \mathrm{~mm}$ in diameter and $80 \mathrm{~mm}$ in length was prepared by water-cooled copper mold suction casting method (WK-II vacuum arc melting furnace), where $T_{g}$ (the glass transition temperature) and $T_{x}$ (the crystallization onset temperature) were $648.1 \mathrm{~K}$ and $734.2 \mathrm{~K}$, respectively. The relationship between the crystallization incubation time of the $\mathrm{BMG}$ and the isothermal holding temperature was confirmed by DSC (Diamond DSC, PerkinElmer Instruments) at a heating rate of $150 \mathrm{~K} \cdot \mathrm{min}^{-1}$, and the viscosity of the BMG supercooled liquid versus the temperature was measured by parallel plate rheometry with TMA (Thermomechanical Analyzer, TMA Q400EM, TA Instruments-Waters $\mathrm{LIC}$ ) under a heating rate of $10 \mathrm{~K} \cdot \mathrm{min}^{-1}$ and a load of $0.9 \mathrm{~N}$ [25].

The hot embossing experiments were conducted with home-made super plasticity microforming equipment, in which the temperature after calibration was controlled in $\pm 3 \mathrm{~K}$ by a Shimaden SR253 program controller. Two stages had been designed in the equipment for the hot embossing process, the preload and load stages. The preload stage was carried out to stabilize the hot embossing system and the load stage was to form microparts. Figure 1(c) showed the sketch of hot embossing process. A BMG feedstock of $3 \mathrm{~mm}$ in diameter and $1 \mathrm{~mm}$ in height was prepared. A silicon wafer with the thickness of $1 \mathrm{~mm}$ was placed beneath the stacked silicon die for padding. The top die moved down under a velocity of $8 \mu \mathrm{m} \cdot \mathrm{s}^{-1}$ before the load was reaching the preset value in the hot embossing process. After that, the redundant flash was removed by mechanical polishing, and the silicon die was etched away in $\mathrm{KOH}$ solution ( $40 \% \mathrm{wt}$ ) under the temperature of $353 \mathrm{~K}$. The appearance of 


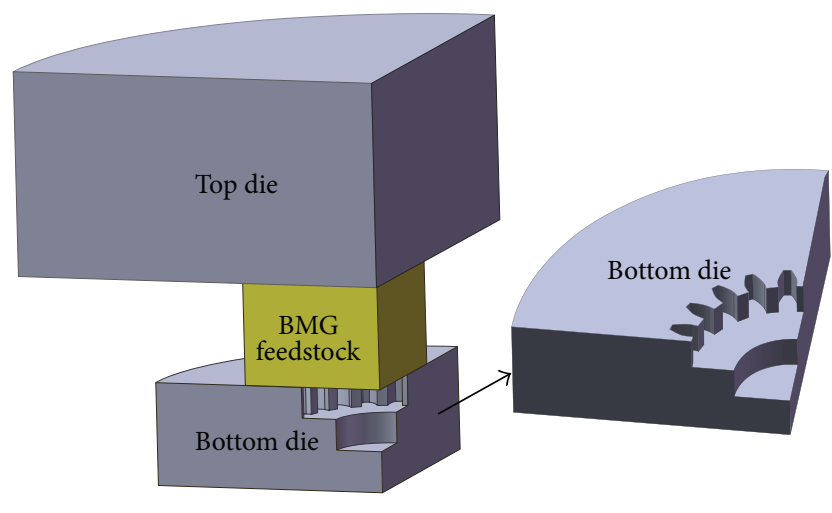

FIGURE 2: Simulation model for hot embossing of BMG microgear.

the obtained micropart was observed by SEM (Scanning Electron Microscopy, FEI Quanta 200), its glassy state was detected with DSC and XRD (X'Pert PRO, PANalytical B.V.), and the mechanical properties were evaluated by nanoindentation tests under a load of $8 \mathrm{mN}$ for $5 \mathrm{~s}$ with the loading and unloading rate of $0.5 \mathrm{mN} \cdot \mathrm{s}^{-1}$ (TI750Ubi system, Hysitron).

\section{Finite Element Simulation}

As mentioned in Figure 1, silicon dies failure will occur under improper loading conditions; thus, the hot embossing load should be optimized and the stress in silicon dies should be maintained in a low level as long as the dies cavity is fully filled by BMG within the limited processing time. DEFORM 3D, finite element simulation software, was employed to investigate the behavior of the BMG in the microdie cavity and the stress in the silicon die. A Newtonian model of $\sigma=$ $\mu \cdot \dot{\varepsilon}$ was adopted, where $\sigma$ is the flow stress, $\dot{\varepsilon}$ is the strain rate, and $\mu$ is the Newtonian viscosity. As shown in Figure 2, a quarter model including a top die, a feedstock, and a bottom die was established to simplify the calculation. The feedstock with $3 \mathrm{~mm}$ in diameter and $1 \mathrm{~mm}$ in height was set as plastic body. The bottom die was a combination of two stacked silicon dies: the first layer was a 20 teeth microgear with $100 \mu \mathrm{m}$ in modulus and $300 \mu \mathrm{m}$ in depth, and the second layer was a shaft with $1 \mathrm{~mm}$ in diameter and $300 \mu \mathrm{m}$ in depth. The pressure and flowing of the BMG supercooled liquid under constant load mode ( $200 \mathrm{~N}$ holding for $120 \mathrm{~s}$ ) and varying load mode $(100 \mathrm{~N}$ holding for $60 \mathrm{~s}$ and then $400 \mathrm{~N}$ holding for $60 \mathrm{~s}$ ) were investigated, where the top and bottom dies were set as rigid bodies. Then, the bottom die was changed to elastic body, and the stress in the bottom silicon dies was studied, in which the Young modulus and Poisson's ratio were set to $186 \mathrm{GPa}$ and 0.27 , respectively.

\section{Results and Discussion}

4.1. Processing Temperature. The BMG feedstock becomes a high viscosity and metastable liquid in the supercooled liquid region and will lose its glassy structure when the processing time exceeds the crystallization incubation time. Table 1 lists the incubation time of $\mathrm{Zr}_{65} \mathrm{Cu}_{17.5} \mathrm{Ni}_{10} \mathrm{Al}_{7.5} \mathrm{BMG}$ obtained by
TABLE 1: Incubation time of $\mathrm{Zr}_{65} \mathrm{Cu}_{17.5} \mathrm{Ni}_{10} \mathrm{Al}_{7.5}$ under different processing temperature.

\begin{tabular}{lcccccc}
\hline Processing temperature (K) & 683 & 693 & 703 & 713 & 723 & 733 \\
\hline Incubation time (min) & 46.3 & 29.6 & 7.92 & 3.57 & 1.67 & 1.00 \\
\hline
\end{tabular}

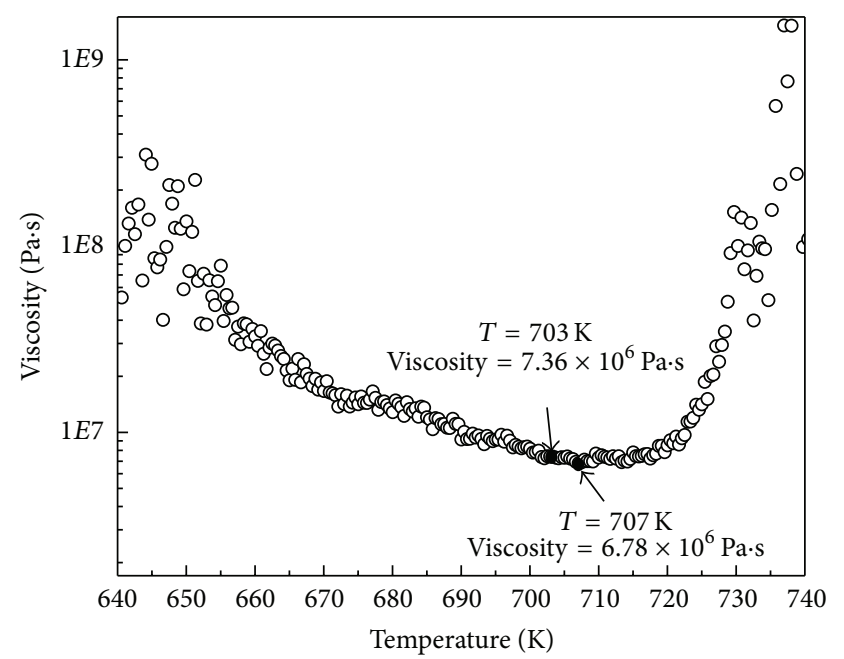

FIGURE 3: Newtonian viscosity of $\mathrm{Zr}_{65} \mathrm{Cu}_{17.5} \mathrm{Ni}_{10} \mathrm{Al}_{7.5}$ BMG versus temperature.

DSC isothermal holding experiments. The incubation time is $46.3 \mathrm{~min}$ at $683 \mathrm{~K}$ and reduces rapidly when the processing temperature increases (1 min @ $733 \mathrm{~K})$. For high processing temperature $(\geq 723 \mathrm{~K})$, the incubation time will be too short to carry out the hot embossing. Thus, we need to choose suitable processing temperature.

The viscosity of $\mathrm{Zr}_{65} \mathrm{Cu}_{17.5} \mathrm{Ni}_{10} \mathrm{Al}_{7.5}$, as shown in Figure 3, is also affected by the temperature and strain rate. Under low strain rate, the BMG supercooled liquid exhibits Newtonian flow and owns best thermoplastic forming ability, where the viscosity is mainly determined by the temperature [26]. When the temperature increases, the viscosity gradually decreases to the minimum value $\left(6.78 \times 10^{6} \mathrm{~Pa} \cdot \mathrm{s}\right.$ at about $707 \mathrm{~K}$ ) in the low temperature zone and then increases in the high temperature zone due to occurrence of crystallization.

Therefore, the processing temperature is the most important parameter for the BMG hot embossing process, as it determines the maximum processing time and the Newtonian viscosity of the BMG supercooled liquid [27]. To enhance the controllability and forming ability, the processing temperature must ensure low viscosity for the supercooled liquid and enough time for the hot embossing process. In this study, the temperature of $703 \mathrm{~K}$ is chosen, in which the maximum processing time reaches $7.92 \mathrm{~min}$ and the viscosity is $7.36 \times 10^{6} \mathrm{~Pa} \cdot \mathrm{s}$.

4.2. Simulation Results. Figure 4 shows the simulation results. The filling process under the constant load mode ( $200 \mathrm{~N}$ for $120 \mathrm{~s}$ ) can be split into three stages. Filling begins in the first stage, in which the maximum pressure and velocity of the supercooled liquid are observed. The macro 


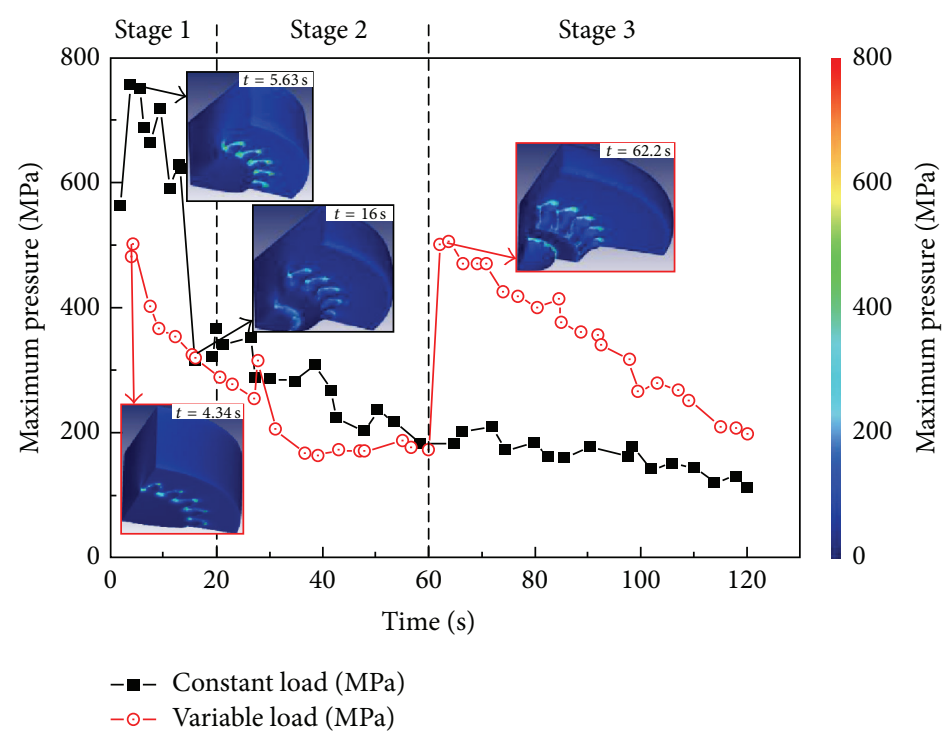

(a)
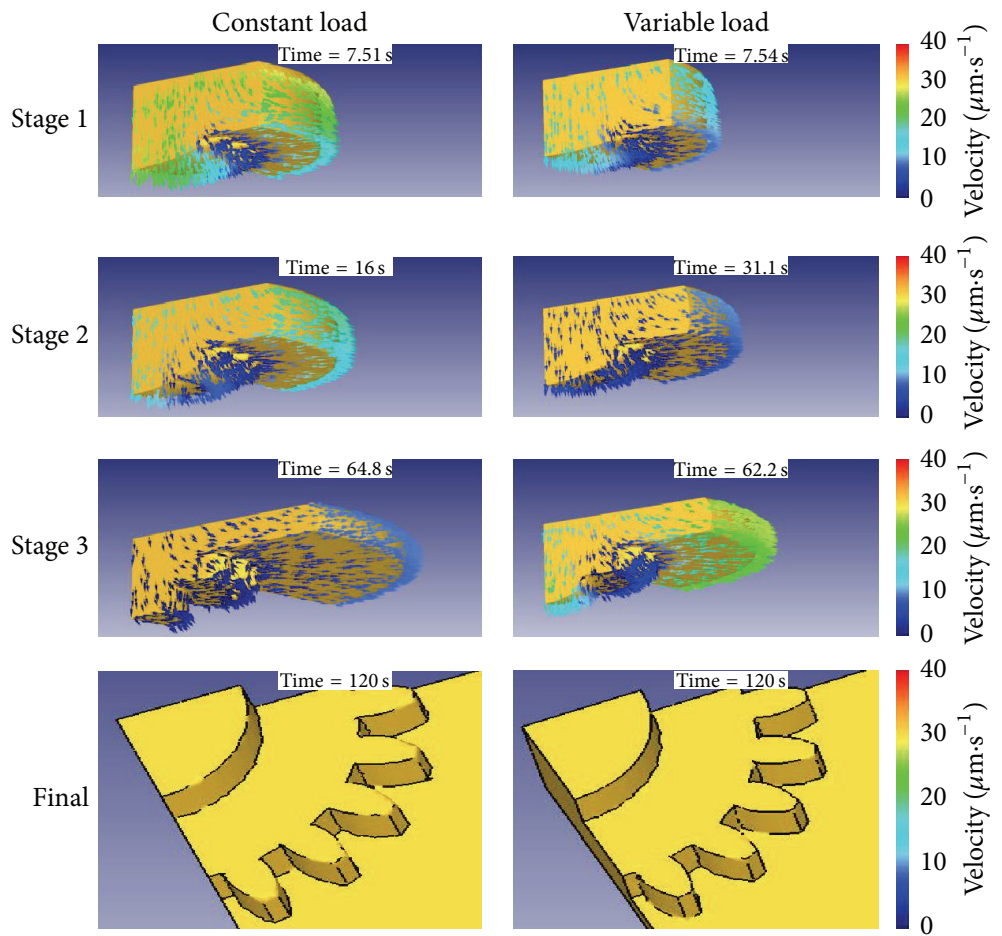

(b)

FIGURE 4: Simulation results: the distribution of pressure (a) and velocity (b) in the BMG supercooled liquid for the two loading modes.

scale structure of the die cavity is filled in the second stage, where both the pressure and the velocity decrease slowly as the filling depth increases. In the third stage, the supercooled liquid flows into the micro scale structures of the die cavity with the pressure and velocity reducing to the minimum values.

Under the varying load mode, the maximum pressure of the supercooled liquid contacting the silicon die is lower (about $500 \mathrm{MPa}$ ) than that under the constant load mode (about $780 \mathrm{MPa}$ ), as shown in Figure 4(a). This illustrates that adopting lower load ( $100 \mathrm{~N}$ for $60 \mathrm{~s})$ in the preload stage will reduce the maximum pressure. When the embossing load is added ( $400 \mathrm{~N}$ for $60 \mathrm{~s}$ ) in the load stage, the pressure is reincreased to $500 \mathrm{MPa}$ and the flowing of the supercooled liquid is accelerated significantly (see Figure 4(b)). The final microparts under the two load modes are shown in Figure 4(b). It can be observed from the magnified velocity distribution that the micropart fabricated with the varying 

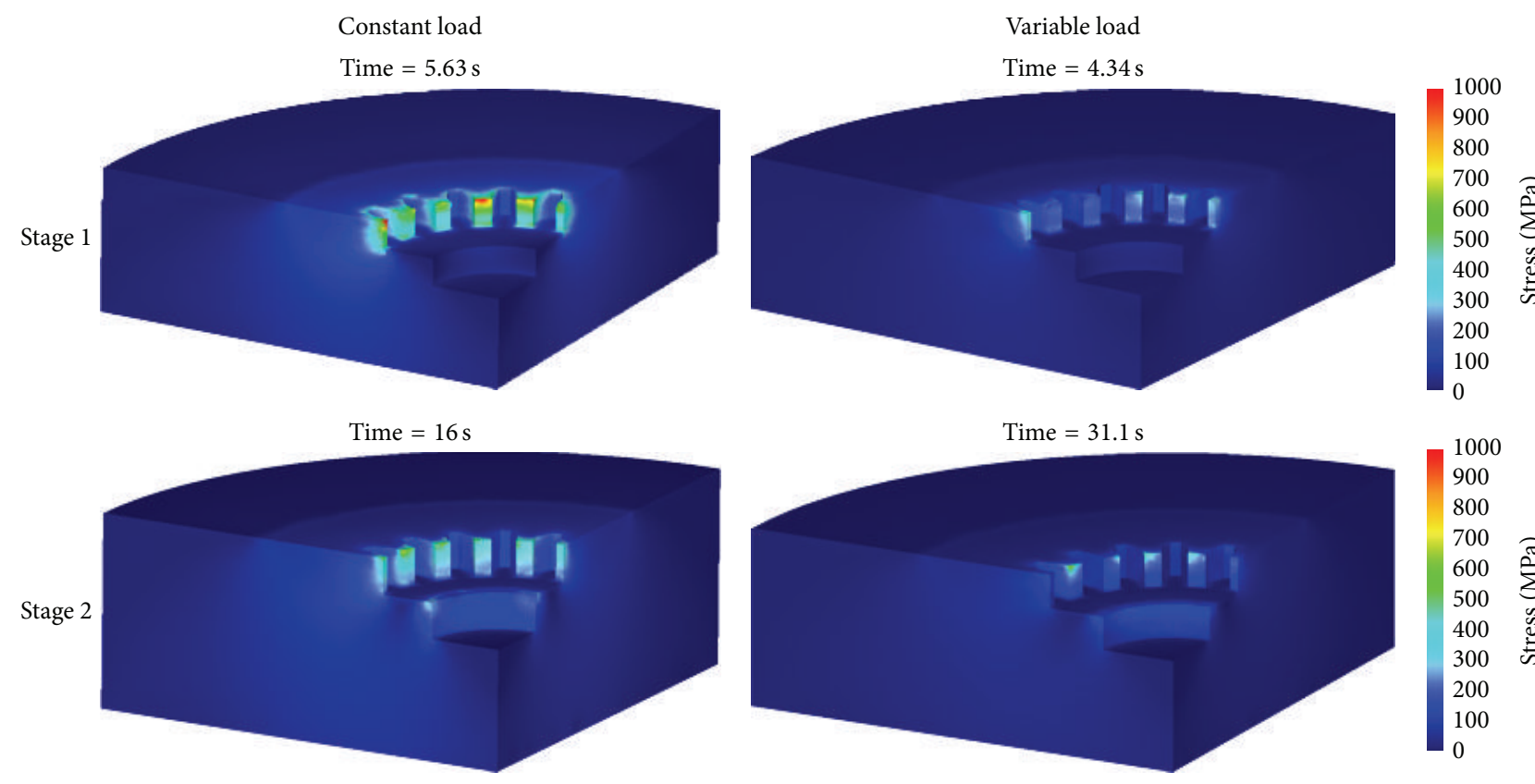

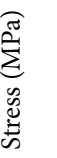
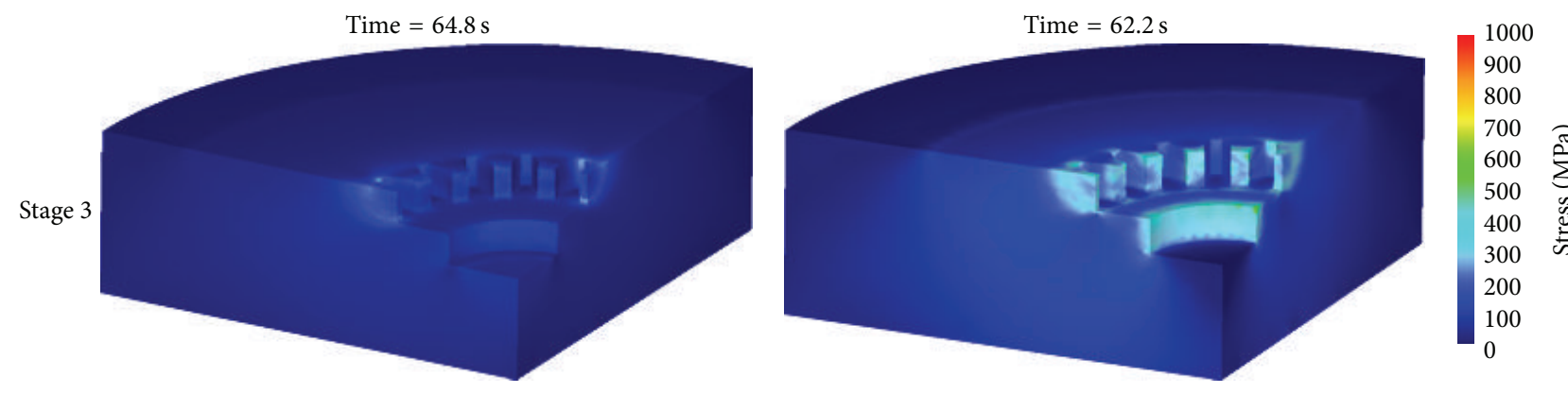

FIGURE 5: The stress distribution in silicon mold for the two load modes.

load mode has more clear edges than that fabricated with the constant load mode. The simulation results demonstrate that under the varying load the maximum pressure during the hot embossing process maintains a lower level, and the micropart will be formed more completely within the same processing time due to the flow acceleration.

Figure 5 shows the stress distribution in the silicon dies. Under the constant load, the stress in the silicon dies concentrates in the gear teeth region (above $500 \mathrm{MPa}$ ) and reaches the highest level (exceeding $1000 \mathrm{MPa}$ ) in the edges of the gear teeth at the first stage. Then, the stress in the gear teeth reduces to about $450 \mathrm{MPa}$ in the second stage and maintains below $150 \mathrm{MPa}$ during the third stage. Under the varying load, the stress in the gear teeth is below $300 \mathrm{MPa}$ at first and increases to the maximum value (about $400 \mathrm{MPa}$ ) after the forming load is added to $400 \mathrm{~N}$ at $62.2 \mathrm{~s}$. As reported in [28], there was a risk of silicon dies failure when the stress exceeded $400 \mathrm{MPa}$. The simulation results suggest that the risk of the silicon dies broken may occur for the constant load mode and can be minished by using the varying load.

4.3. Experimental Result and Micropart Inspection. Figure 6(a) shows the curves of the embossing force versus time for the two load modes. It can be seen that there is a pause at about $110 \mathrm{~N}$ before the embossing force was increasing to $200 \mathrm{~N}$ under the constant load mode. This implies that the force about $110 \mathrm{~N}$ is enough for the supercooled liquid flowing into the dies cavity in the first stage. The silicon dies keep unbroken under the varying load in the experiments. The insets of Figure 6(a) display the microscale structures obtained by these two load modes. We can find that the micropart fabricated under the varying load has more distinct edges than that under the constant load. Figure 6(b) shows the SEM image of the whole 3D BMG micropart embossed under the varying load, demonstrating a good appearance. These are all in agreement with the simulation results.

Figure 7(a) shows the DSC curves of the hot embossed micropart and the as-cast BMG feedstock under the heating rate of $20 \mathrm{~K} / \mathrm{min}$. The distinct glass transition and crystallization heat of the micropart are in accordance with the amorphous alloy, where $T_{g}$ and $T_{x}$ are $645.7 \mathrm{~K}$ and $730.2 \mathrm{~K}$, and the crystallization heat is $30.39 \mathrm{~J} \cdot \mathrm{g}^{-1}$, lower than those of the as-cast BMG. The XRD curves of the as-cast BMG and micropart are shown in Figure 7(b). The two curves show similar patterns with a broad diffuse peak typical for the presence of noncrystalline phases rather than sharp 


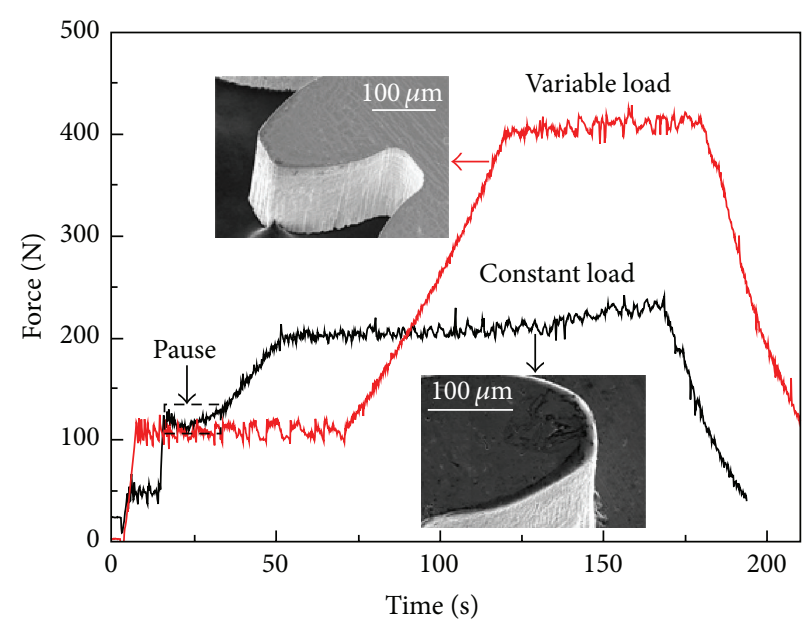

(a)

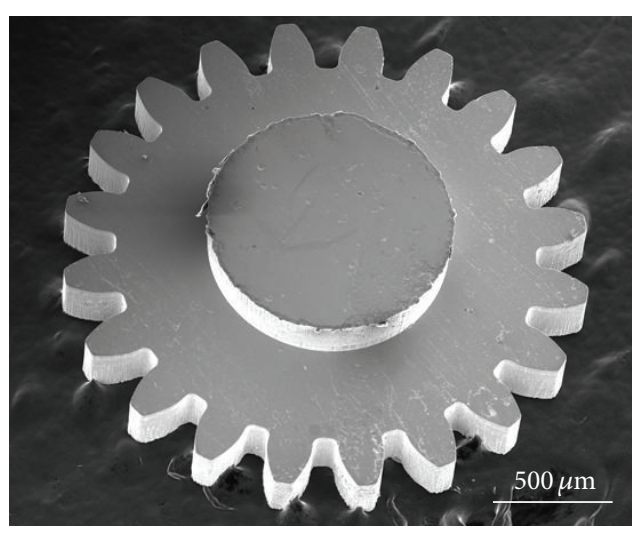

(b)

FIGURE 6: The experimental results. (a) True force curves for the two load modes with the details of the microgear teeth in the insets, (b) SEM image of the microgear formed under the varying load.

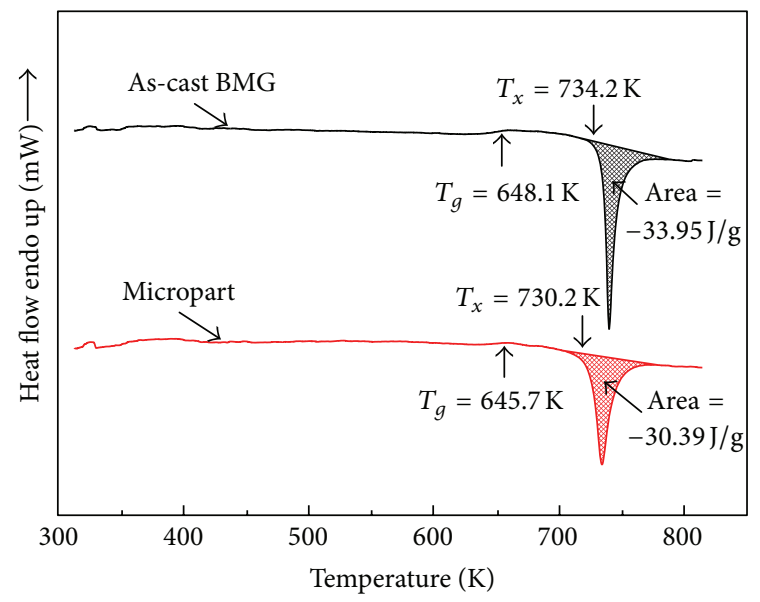

(a)

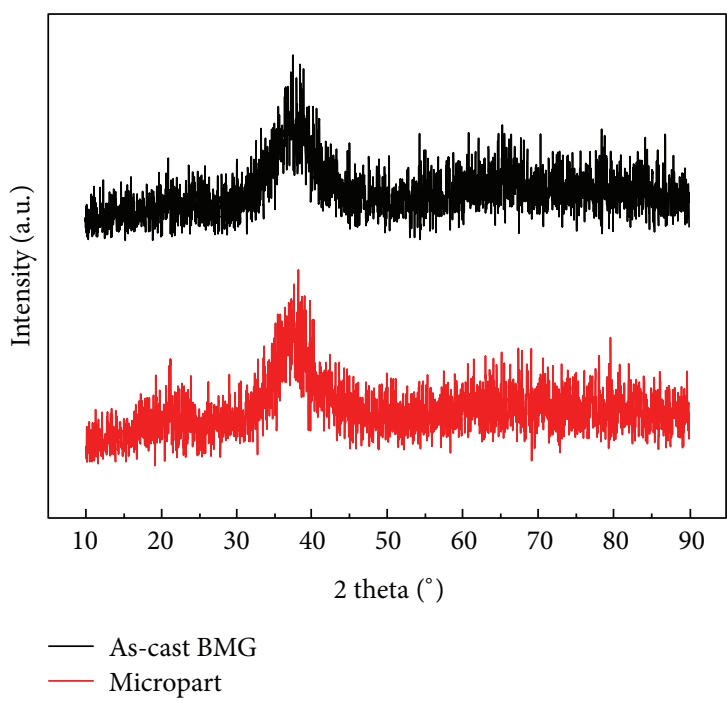

(b)

FIGURE 7: The results of DSC (a) and XRD (b) for the as-cast BMG and microgear.

diffraction peaks, confirming that no large size crystals occur in the micropart during the hot embossing process.

The nanohardness and Young's modulus for the micropart and as-cast BMG feedstock are displayed in Figures 8(a) and $8(\mathrm{~b})$, where the averaged nanohardness and Young's modulus of the microgear are $5.52 \mathrm{GPa}$ and $89.28 \mathrm{GPa}$, very close to those of the as-cast BMG rod (5.48 GPa and 88.07 GPa).

\section{Conclusions}

The fabrication of $\mathrm{Zr}_{65} \mathrm{Cu}_{17.5} \mathrm{Ni}_{10} \mathrm{Al}_{7.5}$ BMG three-dimensional microgear by hot embossing process with the stacked silicon dies is investigated. The processing temperature of $703 \mathrm{~K}$ is chosen, in which the BMG exhibits enough processing time $(7.92 \mathrm{~min})$ and good fluidity $\left(7.36 \times 10^{6} \mathrm{~Pa} \cdot \mathrm{s}\right.$ in viscosity). The finite element simulation results reveal that under the varying load mode ( $100 \mathrm{~N}$ for $60 \mathrm{~s}$ and then $400 \mathrm{~N}$ for $60 \mathrm{~s}$ ) the maximum pressure of the BMG supercooled liquid will maintain a lower level and the edges of the micropart can be formed more clear than that under the constant load mode ( $200 \mathrm{~N}$ for $120 \mathrm{~s}$ ). The experimental results agree well with the simulation. The silicon dies failure is not observed under the varying load, and the formed microgear has a good appearance. The glassy state is preserved after the hot embossing process, while the structure relaxation happens in the BMG micropart. The mechanical properties of the microgear (with the nanohardness $5.52 \mathrm{GPa}$ and Young's modulus $89.28 \mathrm{GPa}$ ) are very close to those of the as-cast BMG feedstock (with the nanohardness $5.48 \mathrm{GPa}$ and Young's modulus $88.07 \mathrm{GPa}$ ). These prove that the durability 


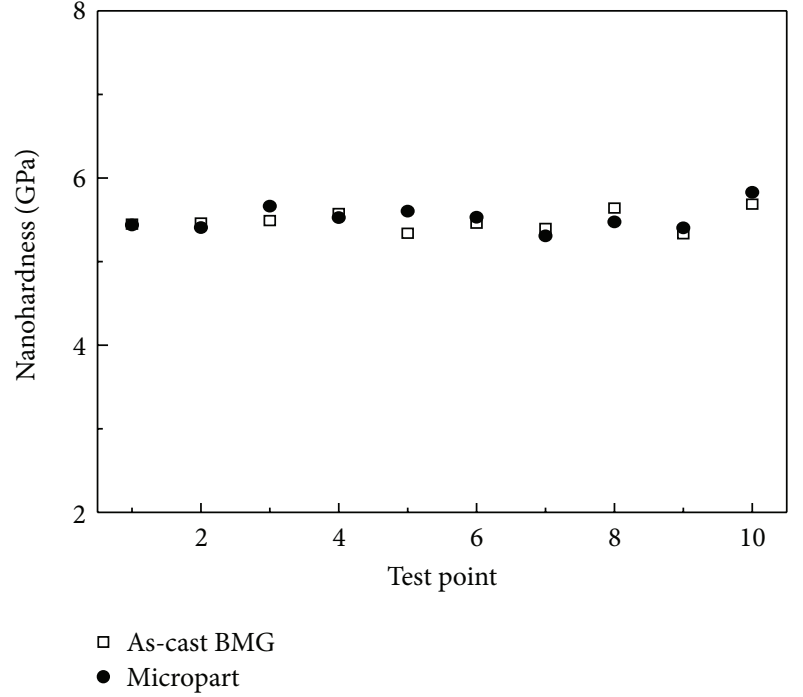

(a)

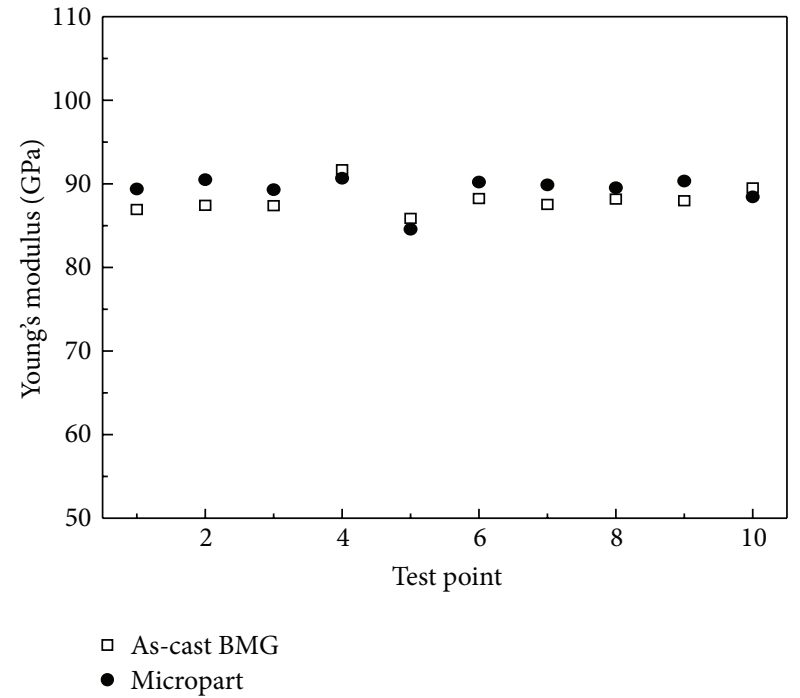

(b)

FIGURE 8: Nanohardness (a) and Young's modulus (b) of the as-cast BMG feedstock and microgear.

of the stacked silicon dies can be enhanced and the threedimensional BMG microparts with good appearance and mechanical properties can be fabricated successfully by using the varying load mode in the hot embossing process.

\section{Conflict of Interests}

The authors declare that there is no conflict of interests regarding the publication of this paper.

\section{Acknowledgments}

The authors gratefully acknowledge the financial supports by the National Key Basic Research Special Fund of China (Grant no. 2015CB057205), the National Natural Science Foundation of China (Grant nos. 51175211 and 51222508), and the Program for Changjiang Scholars and Innovative Research Team in University (no. IRT13017).

\section{References}

[1] M. M. Trexler and N. N. Thadhani, "Mechanical properties of bulk metallic glasses," Progress in Materials Science, vol. 55, no. 8, pp. 759-839, 2010.

[2] A. Inoue and N. Nishiyama, "New bulk metallic glasses for applications as magnetic-sensing, chemical, and structural materials," MRS Bulletin, vol. 32, no. 8, pp. 651-658, 2007.

[3] Y. J. Huang, J. Shen, and J. F. Sun, "Bulk metallic glasses: smaller is softer," Applied Physics Letters, vol. 90, no. 8, Article ID 081919, 2007.

[4] R. D. Conner, Y. Li, W. D. Nix, and W. L. Johnson, "Shear band spacing under bending of Zr-based metallic glass plates," Acta Materialia, vol. 52, no. 8, pp. 2429-2434, 2004.

[5] J. Schroers and N. Paton, "Amorphous metalalloys," Advanced Materials \& Processes, vol. 164, no. 1, pp. 61-63, 2006.
[6] M. Ishida, H. Takeda, D. Watanabe et al., "Fillability and imprintability of high-strength Ni-based bulk metallic glass prepared by the precision die-casting technique," Materials Transactions, vol. 45, no. 4, pp. 1239-1244, 2004.

[7] Z. Zhu, C. Yi, T. Shi, Y. Gao, C. Wen, and G. Liao, "Fabricating Zr-based bulk metallic glass microcomponent by suction casting using silicon micromold," Advances in Mechanical Engineering, vol. 6, Article ID 362484, 8 pages, 2014.

[8] Y. Yokoyama, E. Mund, A. Inoue, and L. Schultz, "Cap casting and enveloped casting techniques for $\mathrm{Zr}_{55} \mathrm{Cu}_{30} \mathrm{Ni}_{5} \mathrm{Al}_{10}$ glassy alloy rod with $32 \mathrm{~mm}$ in diameter," Journal of Physics: Conference Series, vol. 144, no. 1, Article ID 012043, 2009.

[9] J. W. Qiao, Y. Zhang, Z. L. Zheng, J. P. He, and B. C. Wei, "Synthesis of plastic Zr-based bulk metallic glass matrix composites by the copper-mould suction casting and the Bridgman solidification," Journal of Alloys and Compounds, vol. 477, no. 1-2, pp. 436-439, 2009.

[10] B. Chen, T. Shi, M. Li, Z. Zhang, Z. Zhu, and G. Liao, "Laser welding of $\mathrm{Zr}_{41} \mathrm{Ti}_{14} \mathrm{Cu}_{12} \mathrm{Ni}_{10} \mathrm{Be}_{23}$ bulk metallic glass: experiment and temperature field simulation," Advanced Engineering Materials, vol. 15, no. 5, pp. 407-413, 2013.

[11] K. C. Chan, L. Liu, and J. F. Wang, "Superplastic deformation of $\mathrm{Zr}_{55} \mathrm{Cu}_{30} \mathrm{Al}_{10} \mathrm{Ni}_{5}$ bulk metallic glass in the supercooled liquid region," Journal of Non-Crystalline Solids, vol. 353, no. 32-40, pp. 3758-3763, 2007.

[12] A. Reger-Leonhard, M. Heilmaier, and J. Eckert, "Newtonian flow of $\mathrm{Zr}_{55} \mathrm{Cu}_{30} \mathrm{Al}_{10} \mathrm{Ni}_{5}$ bulk metallic glassy alloys," Scripta Materialia, vol. 43, no. 5, pp. 459-464, 2000.

[13] J. Schroers, Q. Pham, and A. Desai, "Thermoplastic forming of bulk netallic glass-a technology for MEMS and microstructure fabrication," Journal of Microelectromechanical Systems, vol. 16, no. 2, pp. 240-247, 2007.

[14] J. J. He, N. Li, N. Tang, X. Y. Wang, C. Zhang, and L. Liu, "The precision replication of a microchannel mould by hotembossing a Zr-based bulk metallic glass," Intermetallics, vol. 21, no. 1, pp. 50-55, 2012. 
[15] C. T. Pan, T. T. Wu, Y. C. Chang, and J. C. Huang, "Experiment and simulation of hot embossing of a bulk metallic glass with low pressure and temperature," Journal of Micromechanics and Microengineering, vol. 18, no. 2, Article ID 025010, 2008.

[16] J. F. Sun, Y. J. Huang, J. Shen, G. Wang, and D. G. McCartney, "Superplastic formability of a $\mathrm{Zr}-\mathrm{Ti}-\mathrm{Ni}-\mathrm{Cu}-\mathrm{Be}$ bulk metallic glass," Journal of Alloys \& Compounds, vol. 415, no. 1-2, pp. 198203, 2006.

[17] G. Wang, J. Shen, J. F. Sun et al., "Superplasticity and superplastic forming ability of a $\mathrm{Zr}-\mathrm{Ti}-\mathrm{Ni}-\mathrm{Cu}-\mathrm{Be}$ bulk metallic glass in the supercooled liquid region," Journal of Non-Crystalline Solids, vol. 351, no. 3, pp. 209-217, 2005.

[18] J. Schroers, "Processing of bulk metallic glass," Advanced Materials, vol. 22, no. 14, pp. 1566-1597, 2010.

[19] D. Wang, G. Liao, J. Pan et al., "Superplastic micro-forming of $\mathrm{Zr}_{65} \mathrm{Cu}_{17.5} \mathrm{Ni}_{10} \mathrm{Al}_{7.5}$ bulk metallic glass with silicon mold using hot embossing technology," Journal of Alloys \& Compounds, vol. 484, no. 1-2, pp. 118-122, 2009.

[20] D. J. Browne, D. Stratton, M. D. Gilchrist, and C. J. Byrne, "Bulk metallic glass multiscale tooling for molding of polymers with micro to nano features: a review," Metallurgical \& Materials Transactions A: Physical Metallurgy and Materials Science, vol. 44, no. 5, pp. 2021-2030, 2013.

[21] X. Zhang, J. Ma, R. Bai, Q. Li, B. Sun, and C. Shen, "Polymer micro hot embossing with bulk metallic glass mold insert," Advanced Materials Research, vol. 510, pp. 639-644, 2012.

[22] J. Bardt, N. Mauntler, G. Bourne, T. L. Schmitz, J. C. Ziegert, and W. G. Sawyer, "Metallic glass surface patterning by micromolding," in Proceedings of the ASME International Mechanical Engineering Congress and Exposition (IMECE '05), pp. 11231129, ASME, November 2005.

[23] J. Böhm, A. Schubert, T. Otto, and T. Burkhardt, "Micrometalforming with silicon dies," Microsystem Technologies, vol. 7, no. 4, pp. 191-195, 2001.

[24] J. A. Bardt, G. R. Bourne, T. L. Schmitz, J. C. Ziegert, and W. G. Sawyer, "Micromolding three-dimensional amorphous metal structures," Journal of Materials Research, vol. 22, no. 2, pp. 339343, 2007.

[25] J. Wang, "Glass viscosity and structural relaxation by parallel plate rheometry using a thermo-mechanical analyser," Materials Letters, vol. 31, no. 1-2, pp. 99-103, 1997.

[26] N. Li, Y. Chen, M. Q. Jiang et al., "A thermoplastic forming map of a Zr-based bulk metallic glass," Acta Materialia, vol. 61, no. 6, pp. 1921-1931, 2013.

[27] E. B. Pitt, G. Kumar, and J. Schroers, "Temperature dependence of the thermoplastic formability in bulk metallic glasses," Journal of Applied Physics, vol. 110, no. 4, Article ID 043518, 2011.

[28] D. Y. R. Chong, W. E. Lee, B. K. Lim, J. H. L. Pang, and T. H. Low, "Mechanical characterization in failure strength of silicon dice," in Proceedings of the 9th Intersociety Conference on Thermal and Thermomechanical Phenomena in Electronic Systems (ITHERM '04), vol. 2, pp. 203-210, Las Vegas, Nev, USA, June 2004. 

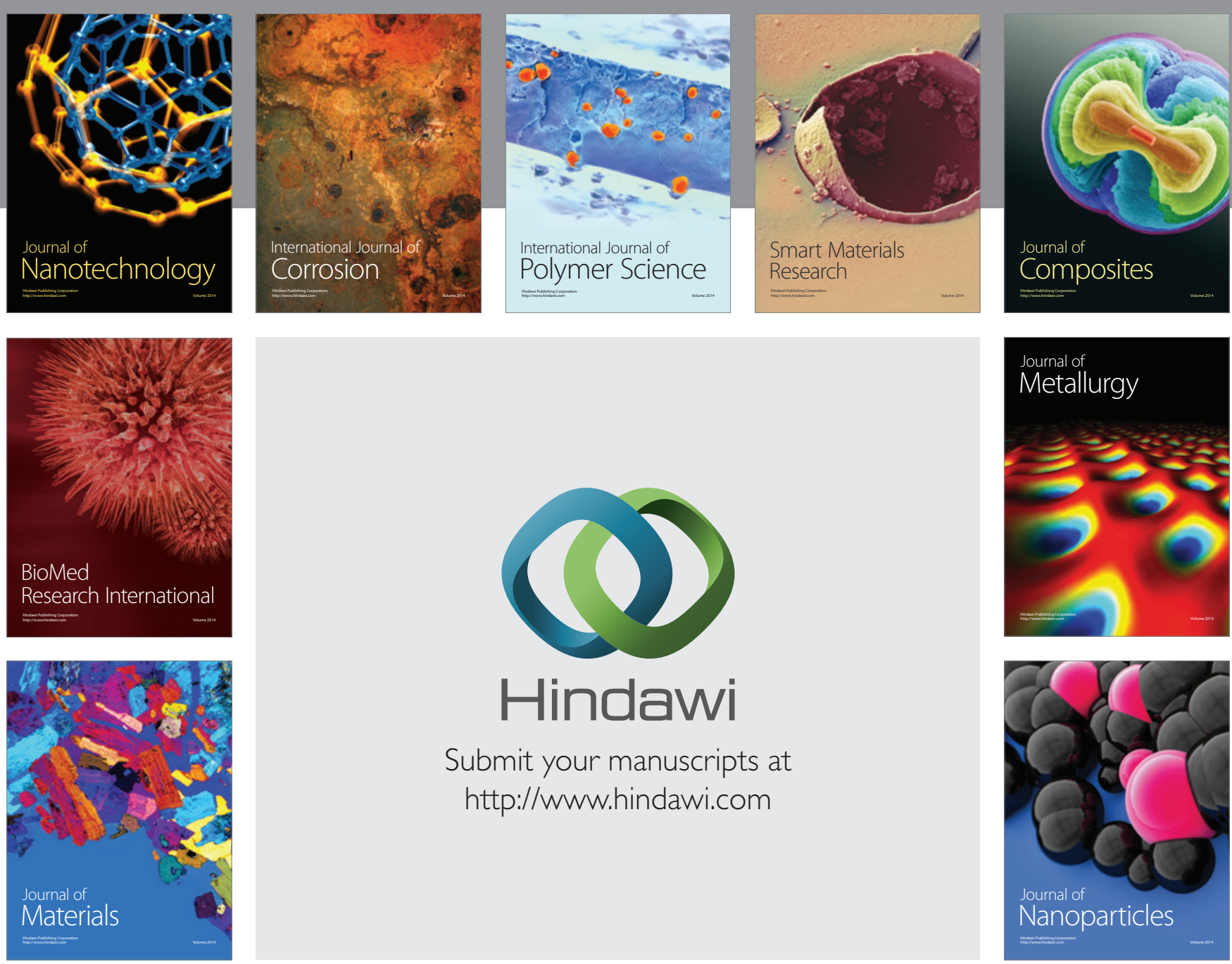

Submit your manuscripts at http://www.hindawi.com
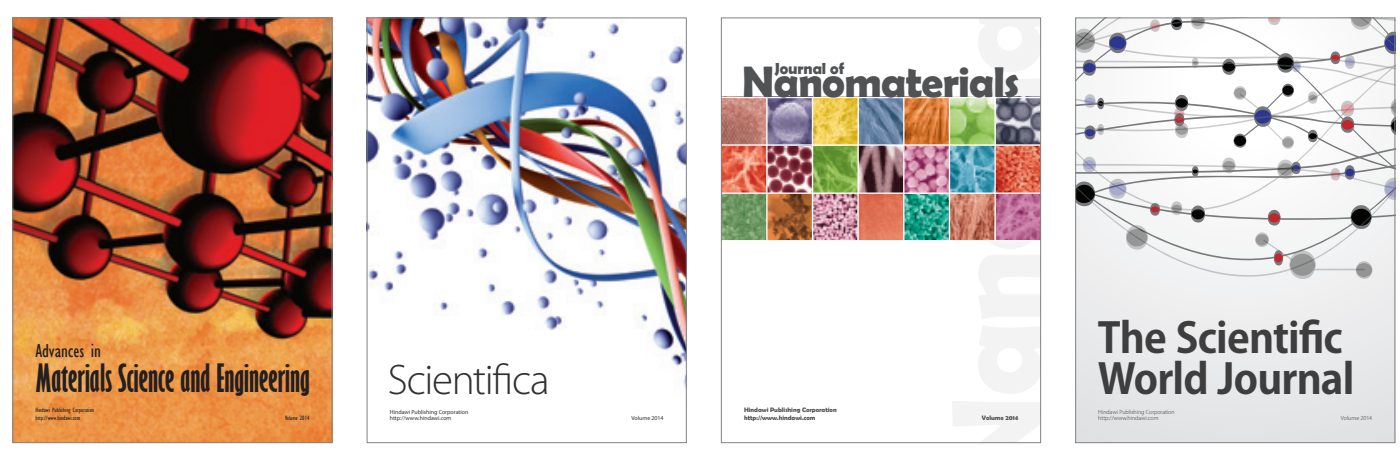

\section{The Scientific World Journal}
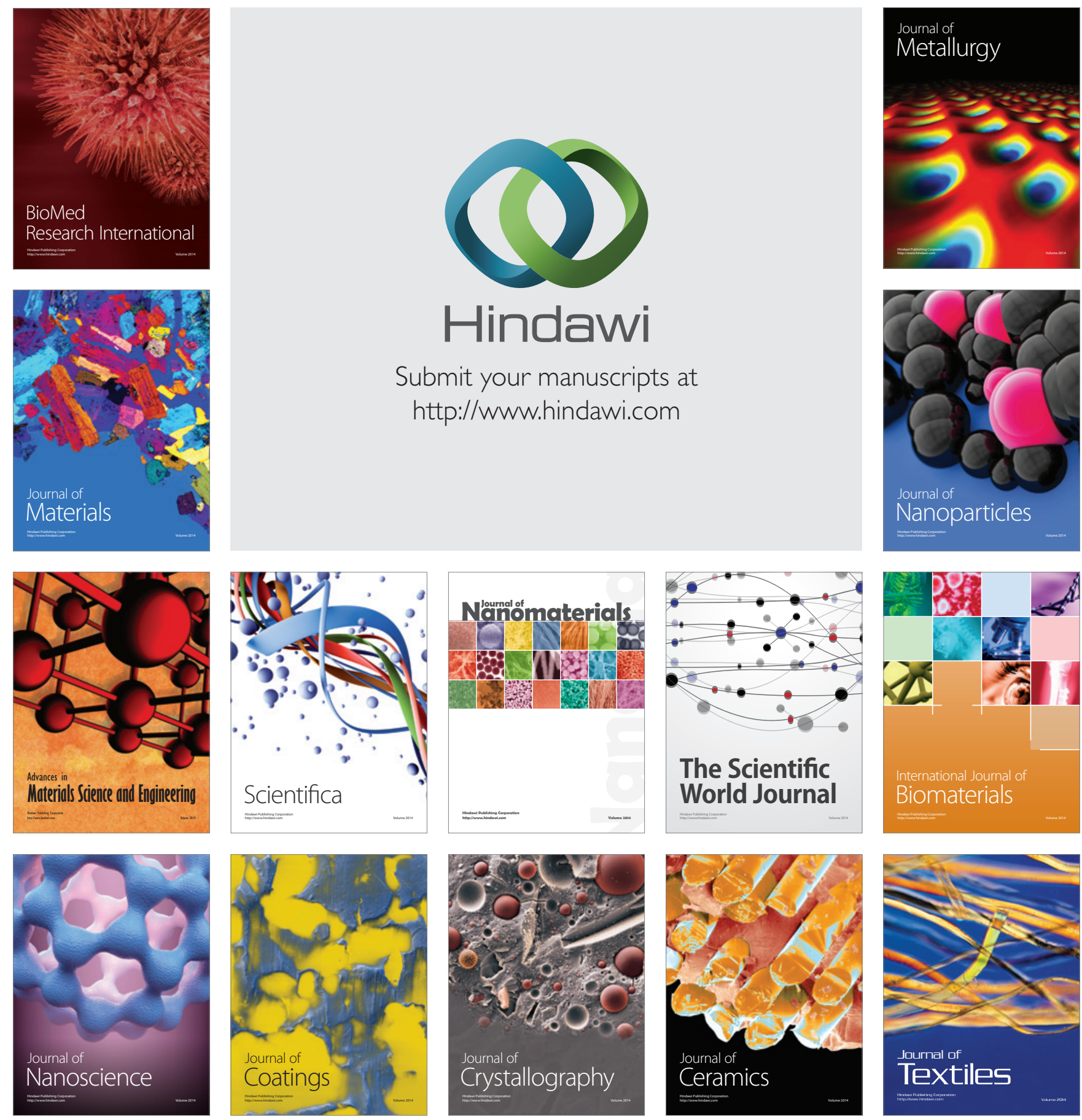